\title{
Evolution of Self-assembled Nanostructure in Glass
}

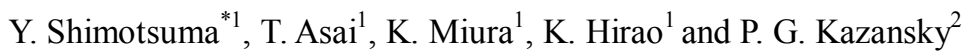 \\ ${ }^{* 1}$ Department of Material Chemistry, Graduate School of Engineering, Kyoto University, Kyoto, \\ 615-8510, Japan \\ E-mail:yshimo@func.mc.kyoto-u.ac.jp \\ ${ }^{2}$ Optoelectronics Research Centre, University of Southampton, SO17 1BJ, United Kingdom
}

\begin{abstract}
We experimentally verify linearly polarized intense ultrashort pulses can control the direction of local optical anisotropy in homogeneous medium based on the quantum interference between photon and electron plasma. Self-assembled nanostructure, which exhibits form-birefringence, has initially evolved from residual birefringence originated from internal stress distribution.
\end{abstract}

DOI: 10.2961/jlmn.2012.03.0019

Keywords: femtosecond laser, self-organization, nanostructure, birefringence, glass

\section{Introduction}

When an intense femtosecond laser pulse is focused into transparent material, e.g. silica glass, high-order nonlinear absorption allows the energy to be deposited predominantly within the focal volume, producing a local permanent refractive-index modification [1]. Although the process of energy absorption is now well understood, little is known about the actual formation of the directly written structures [2]. Depending on the level of laser intensity, one can induce any of three qualitatively different types of modification or damage in silica glass: low intensity induces a smooth positive refractive-index change relative to the unprocessed material (type-I); intermediate intensity results in birefringent regions (type-II); and at high intensity the damage consists of voids embedded into the glass (typeIII). When the induced refractive index change is positive, type-I modification has been used to achieve waveguides and couplers $[1,3]$, resulting from the structural densification induced by fast cooling after the photoexcitation or shock wave generation [4,5]. In spite of the many previous efforts, the origin and the mechanism of the refractive index change have not been fully elucidated [6]. Whereas type-III void like defects have been exploited for data storage and photonic crystals $[7,8]$, which are derived from an electron-ion plasma triggered by avalanche ionization via inverse bremsstrahlung $[9,10]$. The intermediate type-II regime has received little attention until now but indeed seems to have intriguing properties. Type-II structures were first observed in Ge-doped silica where they show an anisotropic light scattering that is dependent on the plane of polarization of the light [11]. Anisotropic scattering has been interpreted in terms of photoelectrons moving along the direction of the light's polarization, inducing index inhomogeneities. Later uniaxial birefringence imprinted in structures written within fused-silica plates was observed $[12,13]$. Anisotropic reflection was then observed and explained in terms of the formation of a self-organized nanograting within the focal volume [14], and finally the existence of the self-induced nanograting was proved by direct imaging $[15,16]$. During the energy absorption process, tunneling, multiphoton and avalanche ionization produce free electrons within the focus of the ultrashort pulsed laser [17]. The observations suggest that, in a certain intensity range, the interference between an incident light field and an electron plasma wave leads to the formation of nano-sized gratings with a pitch as small as $150 \mathrm{~nm}$ [15]. These periodic nanostructures are ruled in the direction parallel to the polarization of the writing laser and consist of thin regions with a low refractive index characterized by a strong oxygen deficiency [15], surrounded by larger regions with a positive index change, similar to type-I modification [18]. Such a periodic assembly behaves as a uniaxial negative birefringent material which is consistent with a planar form birefringence, whose optical axis is parallel to the direction of the polarization of the writing laser. It has been shown that these self-assembled nanostructures indicate the local refractive index change $(\sim-0.1)$ with respect to the unprocessed material [18], change due to variation of the pulse duration [19]. Embedded micro-reflectors [14], retardation plates [18], and micro-fluidic channels [19] based on these nanostructures have been reported; however the mechanism including dynamics of self-organized nanostructures formation is still not fully understood [20]. Here we experimentally demonstrated evolution of form birefringence (type-II) produced by self-organized nanogratings as a function of interpulse intervals and number of pulses. The polarization-dependent photosensitivity in type-II regions was also discussed.

\section{Experimental}

\subsection{Evolution of form birefringence (type-II) as a func- tion of $\tau_{\text {int }}$ and $N_{\text {pulse }}$}

In the experiments, a mode-locked, regeneratively amplified femtosecond laser pulse (Coherent Inc.; Mira9000Legend), operating at $800 \mathrm{~nm}$ with 70 fs pulse was focused inside a commercially available synthetic fused silica (Shin-Etsu Quartz; VIOSIL-SQ), containing approximately 500 ppm OH, via a microscope objective (Nikon; LU Plan Fluor, $100 \times 0.90$ N.A.) at a depth of about $100 \mu \mathrm{m}$ below the sample surface. The pulse energy was about $1.0 \mu \mathrm{J}$ and the beam power measured after microscope objective was independent on the orientation of light polarization. Repetition rate of the laser was set to $250 \mathrm{kHz}$ corresponding to 
the interpulse interval $\tau_{\text {int }}$ of $4 \mu \mathrm{s}$. The interpulse time $\tau_{\text {int }}$ and the number of pulses $N_{\text {pulse }}$ were controlled by field programmable gate array (National Inst.; FPGA module 7811R) produced the trigger pulses. A series of dots were written in silica with different $\tau_{\text {int }}$ and $N_{\text {pulse }}$. After writing, the modified structures were inspected using optical and polarization (CRi Inc.; LC-Polscope) microscopes. Previously we have observed the periodic nanostructure was induced in the orthogonal direction to the laser light polarization [15]. Such nanograting structures, consisting of periodic oxygen defect regions, are responsible for form birefringence [18]. The direction of slow axis of formbirefringence $\left(a_{\text {slow }}\right)$, which is always perpendicular to the writing light polarization $(E)$, can be controlled by rotating polarization direction[20]

\subsection{Pulse-by-pulse analysis of plasma emission during femtosecond laser irradiation}

To discuss the energy transfer from the laser radiation to the light-matter interaction during femtosecond laser irradiation, we performed pulse-by-pulse analysis of plasma emission during femtosecond laser irradiation. In this measurement, the trigger signal from FPGA is sent to a multichannel spectrometer (Hamamatsu Photonics; PMA11) via a delay pulse generator (Stanford Research; DG535). The emission spectra are taken by the detector at time that trigger signal rise with an acquisition gate width of 1 $\mu$ s (Fig. 1). We have also observed transmission spectroscopy during laser beam irradiation using a multichannel spectrometer. In the characterization, a confocal Raman spectrometer (Tokyo Instruments; Nanofinder 30) was used for structural identification of the irradiated regions. Furthermore, in order to characterize the polarization properties of the modified structures in visible region, we measured the Mueller matrix spectrum by using spectropolarimeter (Tokyo Instruments; Poxi-spectra).

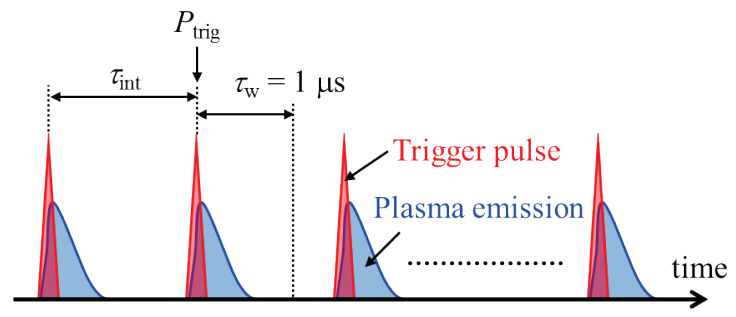

Fig. 1 Pulse diagrams of pulse-by-pulse analysis of plasma emission. $P_{\text {trig, }}, \tau_{\text {int }}$ and $t_{\mathrm{w}}$ indicate a trigger pulse, a laser interpulse time and an acquisition gate width, respectively.

\section{Results and discussion}

\subsection{Structural characterization of Type-II modification}

Fig. 2 indicates optical micrograph of femtosecond laser induced structure in silica glass with different number of pulses $\left(N_{\text {pulse }}\right)$ for the interpulse intervals $\left(\tau_{\text {int }}\right)$ of $4 \mu \mathrm{s}, 40$ $\mu \mathrm{s}$, and $400 \mu \mathrm{s}$. The pulse energy was $1.0 \mu \mathrm{J}$. The length of the modified region was saturated to about $20 \mu \mathrm{m}$ at 100 pulses irradiation for every interpulse time. It should be noted that the size changes of the modified region with increasing number of pulses were independent of the interpulse interval. Even though it is expected to be stronger heat accumulation produced by higher repetition rate femtosecond laser irradiation, the size of the modified region was smaller than that of other multicomponent glass [21]. This phenomenon could be caused by a low thermal expansion coefficient of silica glass $\left(\sim 5 \times 10^{-7} \mathrm{~K}^{-1}\right)$ [22]. The crossed-nicol images reveal that the form birefringence (type-II modification) was observed on top of modified region after 100 pulses irradiation. Additionally in the case of longer interpulse time, the form birefringence was slightly observed at 50 pulses, indicating that the threshold number of pulses in type-II modification tends to decrease with increasing interpulse time. Such phenomenon could be interpreted in terms of the annihilation of the induced oxygen defect, which is especially dominant in the case of shorter interpulse time corresponding to the higher thermal accumulation effect.

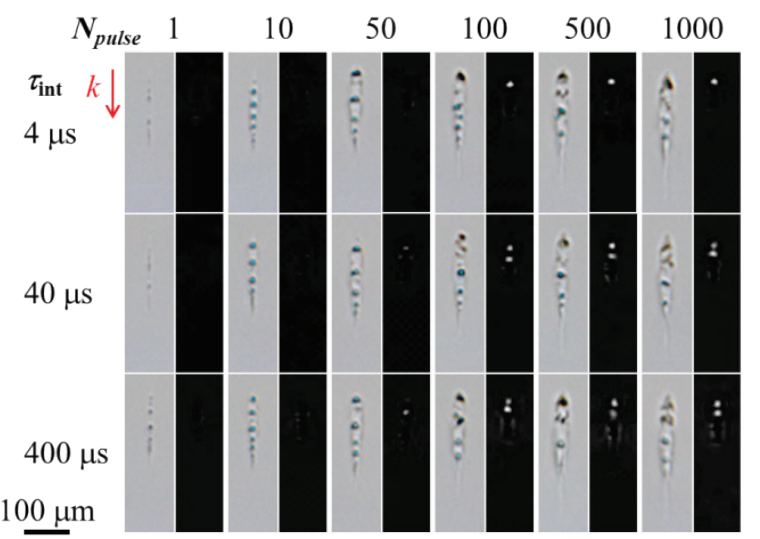

Fig. 2 Cross-sectional optical micrograph of femtosecond laser induced structure in silica glass with different number of pulses $\left(N_{\text {pulse }}\right)$ for various interpulse intervals $\left(\tau_{\text {int }}\right)$ taken with conventional (left) and crossed-nicol (right) configuration. The $k$ arrow indicates the laser propagation direction.
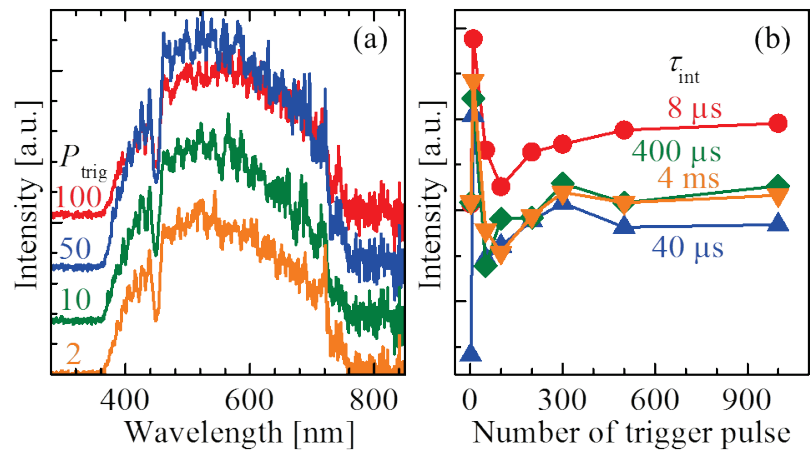

Fig. 3 (a) Pulse-by-pulse plasma emission spectra by changing number of trigger pulse $\left(P_{\text {trig }}\right)$. The acquisition gate width is $1 \mu \mathrm{s}$ (b) The peak intensity profiles of plasma emission ranging from $350 \mathrm{~nm}$ to $750 \mathrm{~nm}$ as a function of the number of trigger pulse for various interpulse times $\left(\tau_{\text {int }}\right)$.

\subsection{Pulse-by-pulse plasma emission}

Fig. 3(a) indicates pulse-by-pulse plasma emission spectra by changing number of trigger pulse $\left(P_{\text {trig }}\right)$. The acquisition gate width is $1 \mu \mathrm{s}$ which is longer than a plasma lifetime and shorter than an interpulse interval $\left(\tau_{\text {int }}\right)$. The broad plasma emission ranging from $350 \mathrm{~nm}$ to $750 \mathrm{~nm}$ was observed regardless of the number of trigger pulse [5]. The drop of emission intensity around $450 \mathrm{~nm}$ was due to the short-pass filter characteristics. The peak intensity profiles of plasma emission ranging from $350 \mathrm{~nm}$ to $750 \mathrm{~nm}$ as a function of the number of trigger pulse for various inter- 
pulse intervals $\left(\tau_{\text {int }}\right)$ were also shown in Fig. 3(b). It was also observed that the plasma emission intensity steeply increased with increasing number of pulse and reached maximum for exposition time below 50 pulses. Subsequent pulses led to a steep decrease of the emission intensity below 100 pulses followed by a slight increase thereafter. It is possible that the observed phenomenon is related to a change of the physical-chemical properties of the modified region after the initial laser irradiation that leads to an alteration involving structural densification, refractive index changes, and/or defect formation.

\subsection{Mueller Matrix Spectrum}

Fig. 4 indicates the spectra of each of the Mueller matrix. The spectra of many of the elements are nearly zero, including M01, M02, M03, M10, M12, M13, M20, M21, M30 and M31. The spectrum of M11 is nearly equal to M00, which indicates the transmission spectrum for sample when unpolarized light is incident. Only elements of M22, M23, M32 and M33 are significantly different from 0 or 1; these are the Mueller matrix elements associated with a linear retarder $\mathrm{M}_{\mathrm{LR}}(\delta)$ of arbitrary retardance $\delta$ oriented with its slow axis at $\theta^{\circ}\left(=0^{\circ}\right.$ or $\left.90^{\circ}\right)$.

$\mathrm{M}_{\mathrm{LR}}(\theta, \delta)$

$$
\propto\left(\begin{array}{cccc}
1 & 0 & 0 & 0 \\
0 & 1 & 0 & 0 \\
0 & 0 & \cos \delta \cos ^{2} 2 \theta+\sin ^{2} 2 \theta & \cos 2 \theta \sin \delta \\
0 & 0 & -\cos 2 \theta \sin \delta & \cos \delta
\end{array}\right)
$$

From these measurements, the modified region also indicates the linear dichroism for the visible light.

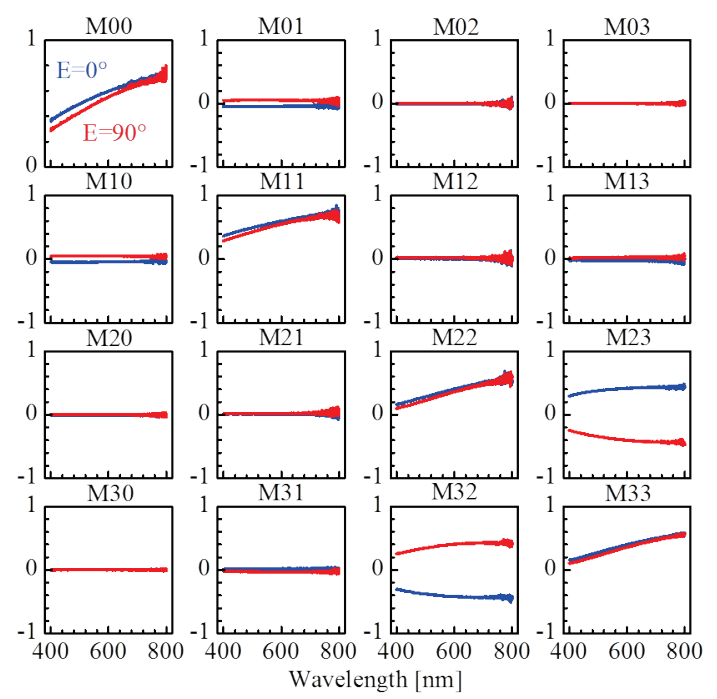

Fig. 4 Mueller matrix spectra of the modified region by using the orthogonally polarized femtosecond pulses (blue: $\mathrm{E}=0^{\circ}$ and red: $90^{\circ}$ ) consists of 16 Mueller matrix element spectra.

\subsection{Photoluminescence from the modified region}

In order to estimate the structural change in silica glass with different $\mathrm{OH}$ concentration induced by femtosecond irradiation, we compared the microscopic fluorescence spectra of femtosecond laser modified regions in a synthetic fused silica (Shin-Etsu Quartz; VIOSIL-SX), containing below $100 \mathrm{ppm} \mathrm{OH}$. In the experiments, microscopic photoluminescence spectra were measured using a CW DPSS laser $(532 \mathrm{~nm})$ as an excitation source at room temperature.
The broad photoluminescence peaks located around $1.9 \mathrm{eV}$ were observed from the laser processed region in the both glass samples (Fig. 5). This peak matches the characteristics of non-bridging oxygen hole center (NBOHC) defects [23]. Despite of the fact that no apparent difference in the size of the modified region was observed between the different $\mathrm{OH}$ concentrations, this broad PL intensity becomes remarkably higher with decreasing $\mathrm{OH}$ concentration. This phenomenon could be interpreted in terms of the dependence of fictive temperature on $\mathrm{OH}$ concentration in silica glass. When femtosecond laser pulses are focused inside the silica glass, most of the pulse energy is deposited into the silica glass sample at the focal volume either through nonlinear absorption or subsequent linear absorption by the plasma, resulting in rapid increase in temperature at the focus. Then many kinds of defects including NBOHC are produced. After irradiation, as the accumulated heat caused by successive pulses diffuses away, the temperature is quickly decreased during several micro seconds [24]. It is known that since the silica glass with a higher $\mathrm{OH}$ concentration exhibits a lower fictive temperature, the silica glass containing a lower $\mathrm{OH}$ concentration requires longer structural relaxation time [25]. In our case, the degree of the structural relaxation of the defects generated in VIOSIL$\mathrm{SX}$ is less compared to that of VIOSIL-SQ. In the case of VIOSIL-SX, the glass structure, including the unrelaxed NBOHC defects, at the higher temperature could be frozen at the cooling process.

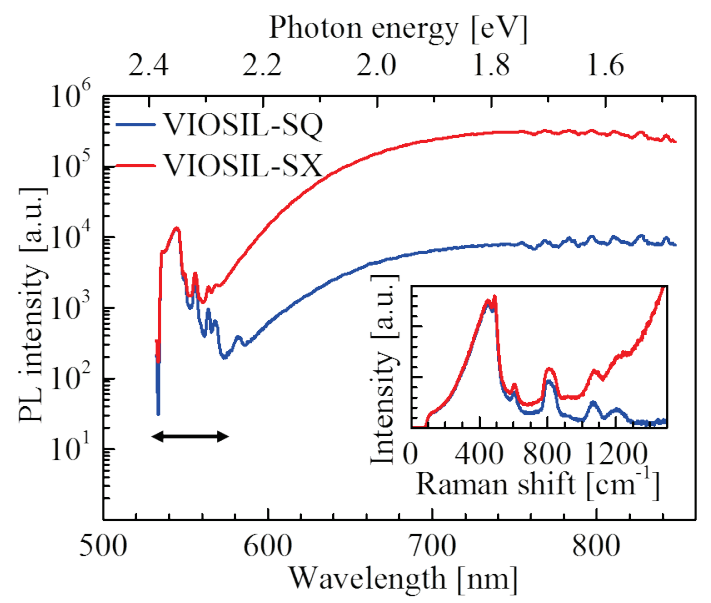

Fig. 5 Microscopic fluorescence spectra of femtosecond laser modified regions in a synthetic fused silica with different $\mathrm{OH}$ concentration. The $250 \mathrm{kHz}$ femtosecond laser pulses with pulse energy of $1 \mu \mathrm{J}$ were focused inside the silica glass samples. Inset shows Raman signals detected on the edge of the fluorescence peak (double-headed arrow).

To understand the effect of the thermal accumulation on the generation of NBOHC defects in VIOSIL-SX glass sample, we have observed the evolution of photoluminescence intensity of $\mathrm{NBOHC}$ defects varying the interpulse time $\tau_{\text {int }}$ and the number of pulse $N_{\text {pulse }}$ at the pulse energy of $1.0 \mu \mathrm{J}$ (Fig. 6). The PL intensity attributed to NBOHC defects initially increases strongly with increasing exposures below 100 pulses and begins to be saturated. Induced NBOHC defects showed tendency to saturate after several hundreds of pulses that could be explained by the competition between creation and annihilation of such defects. In spite of the same pulse energy, the evolution of PL intensity 
is obviously different depending on the polarization direction, especially for the shorter interpulse time corresponding to the higher thermal accumulation effect. For interpulse time $\tau_{\text {int }}$ of $4 \mu$ s and number of pulse above 100 pulses the saturated PL intensity produced by the vertical polarization $\left(\mathrm{E}=90^{\circ}\right)$ was 10 times lower compared with the horizontal polarization $\left(\mathrm{E}=0^{\circ}\right)$, suggesting that horizontal and vertical polarization produces different heating. Let us assume that horizontal polarization produces less heating than vertical. The thermal accumulation effect for horizontal polarization is also less than vertical. In this case the generated NBOHC defects by vertically polarized pulses could be partially annihilated by the recombination of NBOHC and E' centers. Deeper investigation of this phenomenon will be required. The oxygen mobility in glass matrix is generally very slow compared with the optical phenomena. Assuming the temperature of the focal spot reaches $\mathrm{T}=3000^{\circ} \mathrm{C}$, the oxygen diffusion length was calculated by Fick's law:

$$
L_{\mathrm{D}}=2 \sqrt{D_{\mathrm{n}} t}, D_{\mathrm{n}}=D_{0} \exp (-Q / R T)
$$

where $D_{0}\left(=2.6 \times 10^{-4} \mathrm{~m}^{2} / \mathrm{s}\right)$ is maximum diffusion coefficient, $Q$ is the activation energy $(=454 \mathrm{~kJ} / \mathrm{mol})$ and $R$ is gas constant. Since the width of the oxygen defect regions in nanograting structure was $\sim 20 \mathrm{~nm}$, the diffusion time was estimated to be at least $40 \mu \mathrm{s}$ [26].

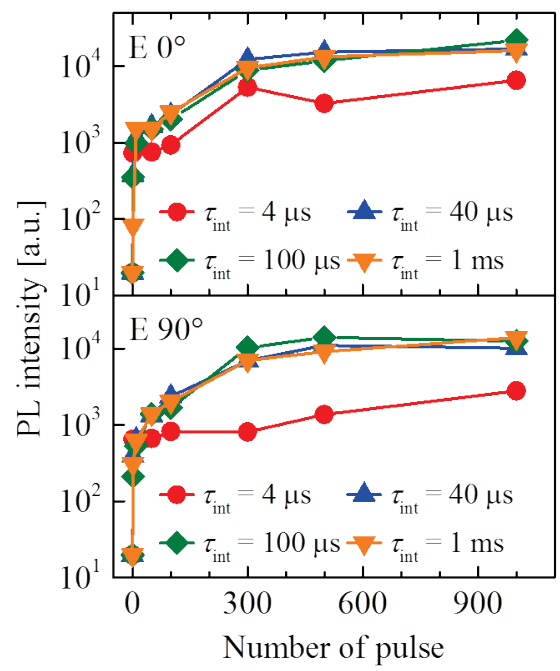

Fig. 6 Evolution of photoluminescence intensity attributed to NBOHC defects for two orthogonal polarizations $\left(\mathrm{E}=0^{\circ}\right.$ and $\left.90^{\circ}\right)$ versus number of pulses with different $\tau_{\text {int }}(4 \mu \mathrm{s}, 40 \mu \mathrm{s}, 100 \mu \mathrm{s}, 1$ $\mathrm{ms})$.

\subsection{Growth mechanism of the optical anisotropy}

In order to reveal the growth mechanism of the anisotropic nanostructure (type-II modification), we have investigated the evolution of birefringence varying repetition rate (or the interpulse time $\tau_{\text {int }}$ ) and exposition (or the number of pulse $N_{\text {pulse }}$ ) at the pulse energy of $1.0 \mu \mathrm{J}$. The Raman spectra of the modified region, taken as a function of $\tau_{\text {int }}$ and $N_{\text {pulse, }}$, have been also observed. To improve the data quality, each Raman spectra have been subtracted a luminescence background corresponding to a $\mathrm{NBOHC}$, and corrected for temperature and frequency dependence of Raman scattering [27]. All the Raman bands have been assigned [28]. For example, the dominant broadband around $450 \mathrm{~cm}^{-1}$ has been attributed to symmetric oxygen vibration of the bent $\mathrm{Si}-\mathrm{O}-\mathrm{Si}$ linkages. The sharp bands peaking at $\sim 490 \mathrm{~cm}^{-1}$ (D1) and $\sim 600 \mathrm{~cm}^{-1}$ (D2) have been attributed to the oxygen breathing modes in four- and three-membered rings of $\mathrm{SiO}_{4}$ tetrahedra, respectively (Fig. 5 inset). These bands are important for obtaining the structural information in the structural modification inside a silica glass, because these bands come from the compaction of silica networks. Here, we focused the intensity change in the D2 band, because this band can be separated more easily from the other bands. The evolution of retardance $\delta$ and intensity of the D2 Raman peak with exposition time was then measured at different interpulse intervals $\tau_{\text {int }}(4,40$, 400, and $4000 \mu \mathrm{s})$ (Fig. 7).
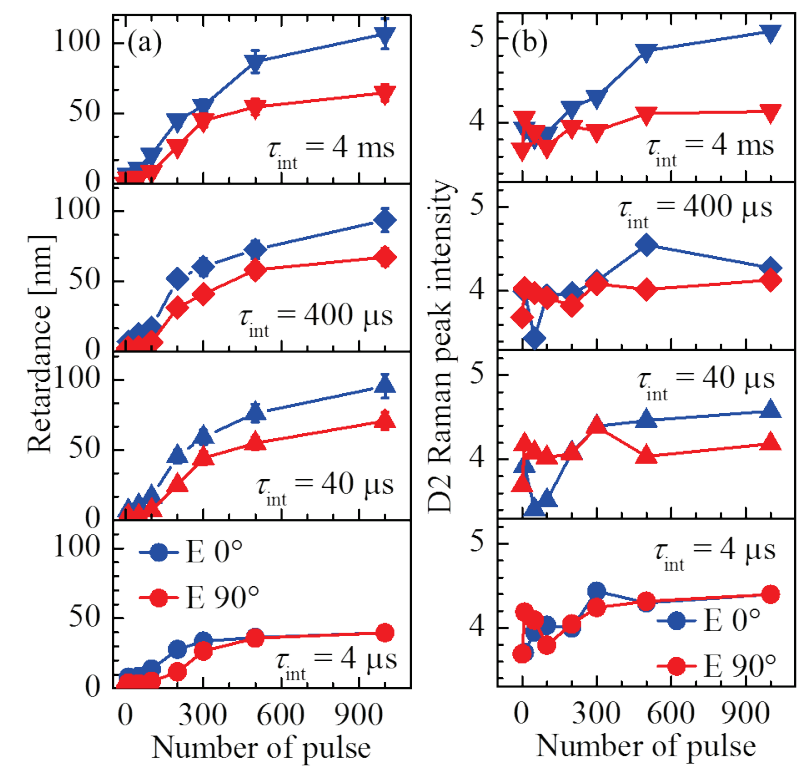

Fig. 7 Evolution of retardance (a) and intensity of the D2 Raman peak (b) for two orthogonal polarizations $\left(\mathrm{E}=0^{\circ}\right.$ and $\left.90^{\circ}\right)$ versus number of pulses with different tint ( $4 \mu \mathrm{s}, 40 \mu \mathrm{s}, 400 \mu \mathrm{s}, 4 \mathrm{~ms})$.
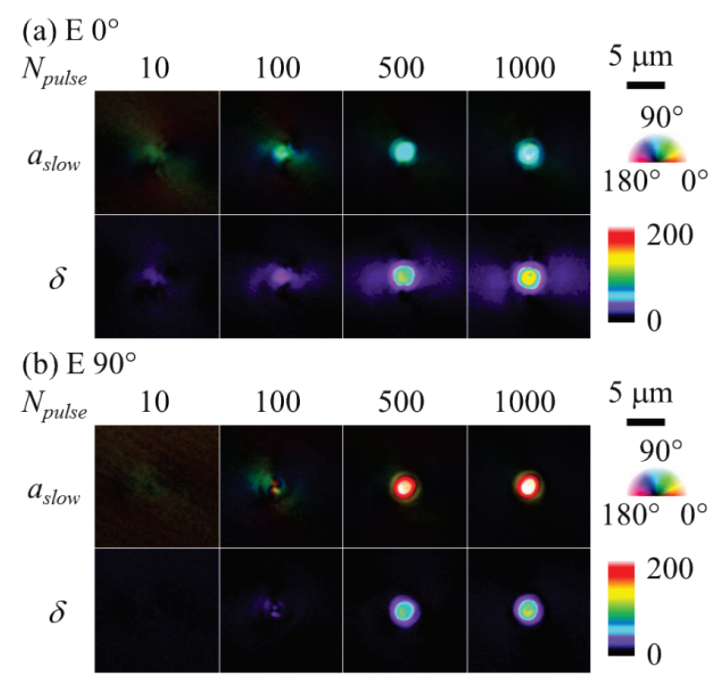

Fig. 8 Micrographs taken with polarization microscope indicating the orientation of slow axis $\left(a_{\text {slow }}\right)$ and the retardance value $(\delta)$ induced by various numbers of pulses at $\tau_{\text {int }}=40 \mu$ s for two orthogonal polarizations of $\mathrm{E}=0^{\circ}$ (a) and $90^{\circ}$ (b).

Micrographs taken with polarization microscope indicating the orientation of slow axis $\left(a_{\text {slow }}\right)$ and the retardance 
value $(\delta)$ induced by various numbers of pulses at $\tau_{\text {int }}$ of 40 $\mu$ s also shown in Fig. 8. In these experiments, we used VIOSIL-SQ glass sample. Only stress birefringence caused by local heating was observed for exposures below 100 pulses. While form birefringence induced by subwavelength periodic structures was detectable only after more than 100 pulses. Induced form birefringence showed tendency to saturate after several hundreds of pulses that could be explained by total depletion of oxygen inside nanograting corrugations. The optimal interpulse time $\tau_{\text {int }}$ to achieve highest retardance value was of $40 \mu \mathrm{s}$, which also corresponds to oxygen diffusion time mentioned above. Alternatively, lower retardance value for $\tau_{\text {int }}<40$ $\mu$ s, could be explained by thermal accumulation [29] which deteriorates quality of the self-assembled nanostructure. These results obviously indicate that the evolution of retardance can be controlled as a function of interpulse time. Such dependence between the retardance, the interpulse time, and the number of pulse can also be seen in the intensity of the D2 Raman peak. Similar changes of the increase in D2 Raman intensity with increasing irradiated pulse energy have been observed, suggesting that an increase in the relative number of the smaller membered silica ring in the glass network leads to an increase in the refractive index. [4]. Furthermore, in spite of the same pulse energy, the evolution of retardance is obviously different depending on the polarization direction, especially for the longer interpulse time corresponding to the lower thermal accumulation effect. For interpulse time $\tau_{\text {int }}$ of $4 \mu \mathrm{s}$ and exposition times bellow 100 pulses the growth speed of the retardance for two perpendicular polarizations $\mathrm{E}=0^{\circ}$ and $90^{\circ}$ was estimated to be $3.6 \times 10^{-2}$ and $1.5 \times 10^{-2} \mathrm{~nm} / \mu \mathrm{s}$, respectively. The lower saturation value of retardance and D2 Raman band intensity produced by the vertically polarized pulses compared with the horizontal polarization could be interpreted in terms of the difference in the internal stress distribution due to local heating. These phenomena indicate that the induced birefringent structure depends on the light polarization and could be related to pulse front tilt [30]. Since the anisotropic sensitivity of an isotropic medium cannot be defined by material structure or its interfaces, however, the experimental results can be derived from intrinsic anisotropy of light-matter interaction involving intense ultrashort light pulses with tilted intensity front. Such heating anisotropy in homogeneous medium could be interpreted in terms of the plasma inhomogeneity originating from the mutual orientation between a light polarization and a pulse front tilt. Indeed we have also observed a weak dependence of the absorbed light power and strong dependence of the hot plasma emission on the light polarization. This reveals that the same amount of absorbed energy can result in different excitations and material modifications.

We propose the following explanation of the dynamics of anisotropic nanostructure self-organization. Once a high free electron density is produced by multiphoton and avalanche ionizations, periodic modulation of electron concentration appears within the pulse width, resulting from the interference between the electric component of the incident light and the produced electron plasma wave. Most electrons are then trapped into deep levels and form the wellknown E' centers within a time scale of $\sim 15$ ps [31]. The electron density modulation is then frozen into permanent material modification. Multiple pulses gradually improve this modulation until total reduction of the oxygen due to diffusion is reached.

\subsection{Interference color change}

Apart from the basic understanding, applying this technique to optical storage, we can achieve the rewritable five dimensions of optical memory (i.e. three dimensions of space (XYZ), polarization direction $(E)$, and phase retardance $(\delta)$ )with a $300 \mathrm{Gbit} / \mathrm{cm}^{3}$ capacity corresponding to the 10 times of the usual $12 \mathrm{~cm}$ BlueRay disk capacity [20]. Additionally, we have also demonstrated the change of interference color by stacking the form birefringence (typeII structure). The phase retardance was tuned by stacking the type-II modified regions with different number of layers spaced by $90 \mu \mathrm{m}$. The retardance value was linearly changed by the number of stacking layers. The interference color under cross-nicol configuration was additively changed by varying the number of stacking layers (Fig. 9).

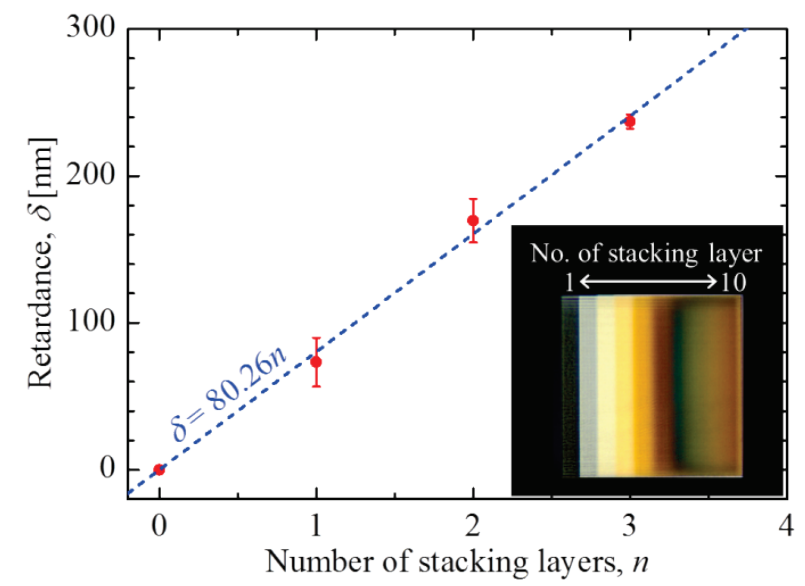

Fig. 9 (a) Phase retardance (d) at $546 \mathrm{nn}$ as a function of the number of stacking layer. Inset shows the interference color of the under cross-nicol configuration for various number of stacking layers.

\section{Conclusion}

In summary, we have experimentally observed evolution of form birefringence (type-II modification). Selforganization of defect structures gradually evolves during multiple pulses irradiation. We have also demonstrated the interference color change by stacking type-II structure in glass. We anticipate that these results will motivate development of a rewritable 5D optical storage.

\section{Acknowledgments and Appendixes}

The authors thank Professor Qiu from Zhejiang University for good suggestion and discussions. This work is partially supported by Grant-in-Aid for Scientific Research (B) and Challenging Exploratory Research.

\section{References}

[1] K. Miura, J. Qiu, H. Inouye, T. Mitsuyu and K. Hirao: Appl. Phys. Lett., 71, (1997) 3329.

[2] C. B. Schaffer, A. Brodeur and E. Mazur: Meas. Sci. Technol., 12, (2001) 1784. 
[3] G. Cerullo, R. Osellame, S. Taccheo, M. Marangoni, D. Polli, R. Ramponi, P. Laporta and S. D. Silverstri: Opt. Lett., 27, (2002) 1938.

[4] J. W. Chan, T. R. Huser, S. H. Risbud and D. M. Krol: Appl. Phys. A, 76, (2003) 367.

[5] W. J. Reichman, J. W. Chan, C. W. Smelser, S. J. Mihailov and D. M. Krol: J. Opt. Soc. Am. B, 24, (2007)1627.

[6] M. Sakakura, M. Terazima, Y. Shimotsuma, K. Miura and K. Hirao: J. Appl. Phys., 109, (2011) 023503.

[7] E. Glezer, M. Milosavljevic, L. Huang, R. J. Finlay, T. H. Her, J. P. Callan and E. Mazur: Opt. Lett., 21, (1996) 2023.

[8] H. Sun, Y. Xu, S. Juodkazis, K. Sun, M. Watanabe, S. Matsuo, H. Misawa and J. Nishii: Opt. Lett., 26, (2001) 325 .

[9] L. Sudrie, A. Couairon, M. Franco, B. Lamouroux, B. Prade, S. Tzortzakis and A. Mysyrowicz: Phys. Rev. Lett., 89, (2002) 186601.

[10]A. Couairon, L. Sudrie, M. Franco, B. Prade and A. Mysyrowicz: Phys. Rev. B, 71, (2005) 125435.

[11]P. G. Kazansky, H. Inouye, T. Mitsuyu, K. Miura, J. Qiu, K. Hirao and F. Starrost: Phys. Rev. Lett., 82, (1999) 2199.

[12]L. Sudrie, M. Franco, B. Prade and A. Mysyrowicz: Opt. Commun., 191, (2001) 333.

[13]E. Bricchi, J. D. Mills, P. G. Kazansky, B. G. Klappauf and J. J. Baumberg: Opt. Lett., 27, (2002) 2200.

[14] J. D. Mills, P. G. Kazansky, E. Bricchi and J. J. Baumberg: Appl. Phys. Lett., 81, (2002) 196.

[15] Y. Shimotsuma, P. G. Kazansky, J. Qiu and K. Hirao: Phys. Rev. Lett., 24, (2003) 2474051.

[16] Y. Shimotsuma, K. Hirao, J. Qiu and P. G. Kazansky: Mod. Phys. Lett. B, 19, (2005) 225.
[17]B. C. Stuart, M. D. Feit, A. M. Rubenchik, B. W. Shore and M. D. Perry: Phys. Rev. Lett., 74, (1995) 2248.

[18]E. Bricchi, B. G. Klappauf and P. G. Kazansky: Opt. Lett., 29, (2004) 119.

[19]C. Hnatovsky, R. S. Taylor, P. P. Rajeev, E. Simova, V. R. Bhardwaj, D. M. Rayner and P. B. Corkum: Appl. Phys. Lett., 87, (2005) 014104.

[20] Y. Shimotsuma, M. Sakakura, P. G. Kazansky, M. Beresna, J. Qiu, K. Miura and K. Hirao: Adv. Mater., 22, (2010) 4039.

[21] Y. Shimotsuma, K. Hirao, J. Qiu and K. Miura: J. Non-Cryst. Solids, 352, (2006) 646.

[22]M. Sakakura, M. Shimizu, Y. Shimotsuma, K. Miura and K. Hirao: Appl. Phys. Lett., 93, (2008) 231112.

[23] J. W. Chan, T. R. Huser, S. H. Risbud and D. M. Krol: Appl. Phys. A, 76, (2003) 367.

[24]M. Sakakura, M. Terazima, Y. Shimotsuma, K. Miura and K. Hirao: Opt. Express, 15, (2007) 16800.

[25] Y. Kokubo, N. Kuzuu, I. Serizawa, L. -H. Zeng and K. Fujii: J. Non-Cryst. Solids, 349, (2004) 38.

[26] J. D. Kalen, R. S. Boyce and J. D. Cawley: J. Am. Ceram. Soc., 74, (1991) 203.

[27]I. Daniel, P. Gillet, B. T. Poe and P. F. McMillan: Phys. Chem. Miner., 22, (1995) 74

[28]P. F. McMillan, B. Piriou and R. Couty: J. Chem. Phys., 81, (1984) 4234.

[29] S. M. Eaton, H. B. Zhang and P. R. Herman: Opt. Express, 13, (2005) 4708.

[30] W. Yang, P. G. Kazansky, Y. Shimotsuma, M. Sakakura, K. Miura and K. Hirao: Appl. Phys. Lett., 93, (2008) 171109.

[31]S. -H. Cho, H. Kumagai and K. Midorikawa: Opt. Commun., 207, (2002) 243.

(Received: June 04, 2012, Accepted: August 30, 2012) 